(C) The Authors 2017. This is an Open Access article, distributed under the terms of the Creative Commons Attribution licence (http:// creativecommons.org/licenses/by/4.0/), which permits unrestricted re-use, distribution, and reproduction in any medium, provided the original work is properly cited.

\title{
A structured literature review on the role of mindfulness, mindful eating and intuitive eating in changing eating behaviours: effectiveness and associated potential mechanisms
}

\author{
Janet M. Warren ${ }^{1 *}$, Nicola Smith ${ }^{2}$ and Margaret Ashwell ${ }^{3}$ \\ ${ }^{1}$ FirstStop Nutrition Ltd, Oxford, UK \\ ${ }^{2}$ Oxford Clinical Psychology, Oxford, UK \\ ${ }^{3}$ Ashwell Associates, Ashwell, UK
}

\section{Abstract}

The role of mindfulness, mindful eating and a newer concept of intuitive eating in modulating eating habits is an area of increasing interest. In this structured literature review, a summary of the current evidence is presented, together with details of interventions undertaken and the tools to measure outcomes. It is broad in scope given the emerging evidence base in this area. The review yielded sixty-eight publications: twenty-three interventions in obese/overweight populations; twenty-nine interventions in normal-weight populations; sixteen observational studies, three of which were carried out in overweight/obese populations. Mindfulness-based approaches appear most effective in addressing binge eating, emotional eating and eating in response to external cues. There is a lack of compelling evidence for the effectiveness of mindfulness and mindful eating in weight management. Mindfulness-based approaches may prevent weight gain. Reduced food intake was seen in some of the studies in overweight and obese populations, but this was less apparent in the studies in normal-weight populations. The evidence base for intuitive eating is limited to date and further research is needed to examine its potential in altering eating behaviours. Mindfulness appears to work by an increased awareness of internal, rather than external, cues to eat. Mindfulness and mindful eating have the potential to address problematic eating behaviours and the challenges many face with controlling their food intake. Encouraging a mindful eating approach would seem to be a positive message to be included in general weight management advice to the public.

\section{Key words: Mindful eating: Intuitive eating: Binge eating: Emotional eating: External eating: Obese: Overweight}

\section{Introduction}

Excessive body weight is one of the most challenging public health issues of our time ${ }^{(1)}$. Our relationship with food is important; a recent UK report on securing a positive 'Food Future' indicated that consumers feel a loss of connection with food that should be preserved ${ }^{(2)}$. Problematic food and eating behaviours, for example binge and emotional eating, have been characterised in obesity. In mindfulness, an individual observes their immediate experience using an open and non-judgemental stance ${ }^{(3)}$. One of the modern-day pioneers of mindfulness is Jon Kabat Zinn who developed the Mindfulness-Based Stress Reduction (MBSR) programme which is used in mainstream medicine internationally, particularly in the management of depression ${ }^{(4)}$. Recent reviews have examined the evidence for mindfulness in the management of pain ${ }^{(5,6)}$, depression ${ }^{(7,8)}$ and psychological well-being ${ }^{(9,10)}$.

In relation to eating behaviours, research began into the use of mindfulness in binge eating with early interventions in the late 1990s when Kristeller \& Hallet ${ }^{(11)}$ undertook a pilot study among obese women with binge eating disorder using Mindfulness-Based Eating Awareness Training (MB-EAT). This intervention was later developed for obese populations with or without binge eating disorder ${ }^{(12)}$. In recent years there has been increased interest in using mindfulness and mindful eating in obese/overweight and normal-weight populations.

Mindful eating consists of making conscious food choices, developing an awareness of physical $v$. psychological hunger and satiety cues ${ }^{(13)}$, and eating healthfully in response to those cues $^{(14)}$. It is conceptualised as being aware of the present moment when one is eating ${ }^{(15)}$, paying close attention to the effect of the food on the senses, and noting the physical and emotional sensations in response to eating ${ }^{(16)}$. There is no universal definition of mindful eating but these principles are consistent in the literature.

Intuitive eating is a discreet, but complementary term, often used interchangeably with mindful eating. Intuitive eating was originally defined by two US Registered Dietitians in $1995^{(17)}$ who described ten aspects of intuitive eating including

Abbreviations: ACT, Acceptance and Commitment Therapy; fMRI, functional MRI; MB-EAT, Mindfulness-Based Eating Awareness Training; MBSR, MindfulnessBased Stress Reduction.

* Corresponding author: Dr Janet Warren, c/o INCA Central Office, Cobweb Buildings, The Lane, Lyford, Wantage, Oxfordshire OX12 0EE, UK, email janet@firststopnutrition.co.uk 
a rejection of 'diets', a discouragement of labelling foods as 'bad', an encouragement to honour hunger, and allow satisfaction with food intake. Both mindful eating and intuitive eating concentrate on internally focused eating ${ }^{(18)}$; intuitive eating, however, does not involve meditation ${ }^{(17)}$.

A number of recent systematic reviews have been published looking at the effectiveness of mindful eating ${ }^{(19)}$, and mindfulness interventions ${ }^{(20)}$ in binge eating, and mindfulness-based interventions for eating disorders and obesity and obesityrelated behaviours ${ }^{(21-23)}$. The three reviews that looked at binge eating showed a positive effect of mindfulness in reducing this maladaptive eating behaviour; the effect sizes were moderate ${ }^{(20)}$. Furthermore, there was evidence of its efficacy in emotional $^{(20,23)}$ and external ${ }^{(23)}$ eating. The role of mindfulness in weight management was less apparent ${ }^{(20,21)}$. The high level of heterogeneity between studies ${ }^{(20)}$ and methodological weakness of many studies ${ }^{(22)}$ were highlighted.

Mindfulness is a key component of interventions based on dialectical behaviour therapy and Acceptance and Commitment Therapy (ACT), which are both third-wave therapies to promote awareness, acceptance and distress tolerance ${ }^{(19)}$.

The aim of this paper is to present the results of a structured literature review looking at the outcomes of mindfulness, including mindful eating and intuitive eating, in changing eating behaviours (including binge eating, emotional eating and eating in response to cues) in healthy populations of any age and weight status. As this is an emerging area of science, the review includes both observational and intervention studies to provide an indication of the direction of future research. The review provides details of the tools used to measure outcomes and the interventions themselves including their theoretical basis. The review also includes a brief summary of potential associated mechanisms that may explain the observed results.

\section{Methodology}

The structured review was carried out combining the following search terms and within the three parameters: population, intervention and outcomes. Search terms were combined with 'or' within parameters, and combined with 'and' across parameters. Only peer-reviewed interventions and observational studies were included.

\section{Population}

Search terms were: healthy population or adult or child or obese or overweight or binge eating or problematic eating or disordered eating or diabetes or diabetic.

\section{Intervention}

Search terms were: mindful eating or mindfulness or mindfulness based intervention or meditation or mindfulness based cognitive behaviour therapy or mindfulness based stress reduction or acceptance based practices or mindful body scan or intuitive eating.

These search terms were also searched in combination with cognitive behaviour/behavior therapy or acceptance and commitment therapy or weight intervention or weight program.

\section{Outcomes}

Search terms were: weight or weight related eating behaviour/ behavior or overeating or eating behaviour/behavior or eating pattern or dietary behaviour/behavior or dietary pattern or snacking behaviour/behavior or diet quality or energy intake or energy balance or meal frequency or external eating or selfregulation or intuitive eating or mindless eating or mindful eating or distracted eating or appetite awareness or hunger recognition or emotional eating or self-efficacy for eating behaviour/behavior or self-efficacy for healthy eating or selfconfidence or wellness or well-being or quality of life.

\section{Databases}

The search was conducted using a University search engine which searched multiple databases: AMED (Alternative MEDicine), ASSIA (Applied Social Sciences Index), BioMed central, BMJ open, British Nursing Index, CINAHL (Nursing and Allied Health), Clinical Evidence, Cochrane Library, Conference Proceedings Citations Index, Ebsco, Medline, NICE, PsycARTICLES, PsycINFO, PubMed, Sage Journals Online and Science Direct.

\section{Inclusion criteria}

Included were papers consistent with the search strategy outlined above in the databases listed, and English articles published until August 2015.

\section{Exclusion criterion}

Excluded were non-English-language papers.

\section{Coding and scoring of results}

For both the intervention and observational studies, results were 'coded' and summarised as follows. A 'positive' code indicated the outcome demonstrated a beneficial effect of mindfulness (for example, decreased binge eating, weight loss, and reduced energy intake). A 'neutral' code indicated that there was no significant effect from the intervention. A mixed code indicated that there were some positive and neutral results. 'Negative' codes indicated that the measured outcome was in an opposite direction to the hypothesis.

Outcomes that were measured in three or more studies were ascribed a percentage score calculated by giving all positive codes one point, negative results were given minus one point, and neutral or mixed codes were given no score. The points were added per outcome, divided by the total number of outcomes and expressed as a percentage.

We acknowledge that this is not a conventional way of summarising the results of the studies and does not account for effect size. However, it provides an overview of findings in the wide range of papers included in the review, and gives an indication of whether or not there is consistency in the literature.

\section{Search strategy results}

The results of the structured literature review are shown in Fig. 1 and resulted in sixty-eight papers. 


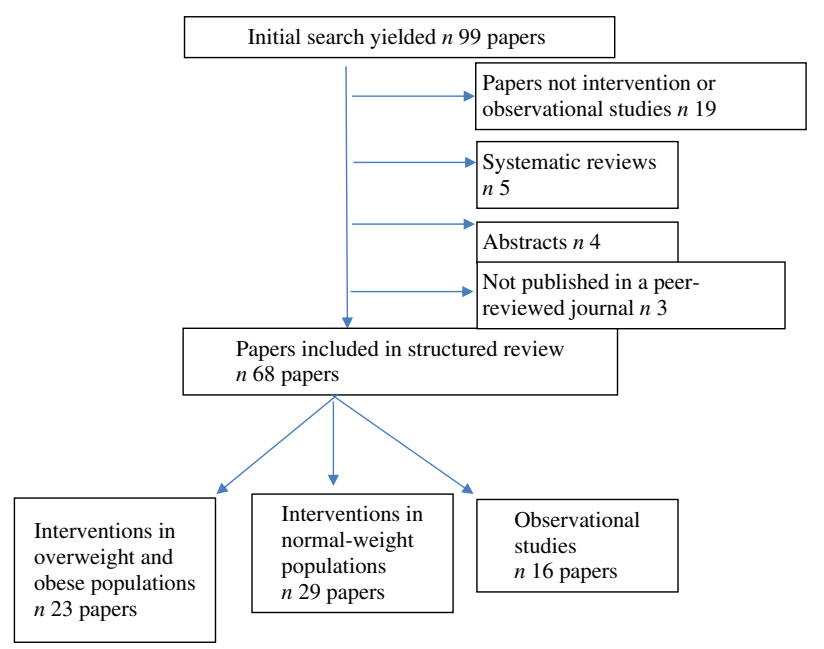

Fig. 1. Literature search results.

\section{Results - intervention studies}

The search yielded twenty-three studies (twenty-four papers) in overweight/obese subjects $^{(11,13,14,16,24-43)}$ and twenty-nine papers describing interventions in normal-weight subjects ${ }^{(15,18,34,44-61)}$.

\section{Characteristics of the intervention studies}

The majority of interventions have taken place in the last 5 years. Most of the interventions in obese/overweight populations were undertaken in female populations, while studies of normalweight populations included more men. Many of the interventions had small sample sizes of fifty or fewer subjects: fourteen of the overweight/obese studies ${ }^{(11,13,24,25,27-29,31-33,37,41,43)}$, and ten of the studies in normal-weight populations ${ }^{(45,47-49,51,54,58-61)}$. Three of the studies in the overweight/obese populations were carried out among children ${ }^{(28,29,40)}$; one of these studies had a sample size of more than 300 but the results of this study were not clearly described ${ }^{(40)}$. The majority of the studies ${ }^{(15)}$ among normal-weight populations were performed with students $^{(15,18,34,46-48,50-53)}$.

\section{Theoretical basis of the interventions in overweight and obese subjects}

Mindfulness-based cognitive therapy/mindfulness action-based cognitive therapy, and mindful eating were the most commonly used approaches and were both used in four studies. Table 1 outlines the details of the theoretical basis of these studies.

\section{Theoretical basis of the interventions in normal-weight populations}

Mindfulness was the most frequently cited basis in the studies among normal-weight subjects and was reported in eight papers which contained thirteen studies ${ }^{(34,46,51,53,56-59)}$. Three papers used mindful eating ${ }^{(15,48,50)}$ and two intuitive eating $^{(18,47)}$. One paper which contained three studies used emotional awareness ${ }^{(55)}$. Cognitive defusion ${ }^{(52)}$ and ACT were each used in one paper ${ }^{(49)}$.
Table 1. Theoretical basis of interventions in overweight and obese subjects ( $n$ 23)

\begin{tabular}{ll}
\hline Theoretical basis & Studies \\
\hline $\begin{array}{l}\text { MBCT or mindfulness action-based therapy } \\
\text { Mindful eating }\end{array}$ & Four studies $^{(25,27,33,40)}$ \\
MBSR & Four studies $^{(28,29,36,43)}$ \\
Mindfulness & Three studies $^{(13,30,54)}$ \\
Mindful eating and MB-EAT & Three studies $^{(11,32,35,44)}$ \\
Mindfulness-based action committed therapy & Three studies $^{(14,16,38,41)}$ \\
Combination of MBSR, MBCT and MB-EAT & Two studies $^{(38,42)}$ \\
Acceptance-based behaviour intervention & One study ${ }^{(31)}$ \\
Acceptance of cravings & One study \\
Intuitive eating & One study \\
\end{tabular}

MBCT, mindfulness-based cognitive therapy; MBSR, Mindfulness-Based Stress Reduction; MB-EAT, Mindfulness-Based Eating Awareness Training.

\section{Intervention length, intensity and scope}

The majority of interventions ( $n$ 18) in the overweight/obese populations consisted of sustained weight-management programmes of medium to high intensity carried out for a medium length of time, i.e. 6 weeks to 4 months. Two studies lasted longer than 4 months and two were for a shorter duration with one study not detailing its intervention length. In many of these studies mindfulness was the sole focus of the intervention which often included mindful eating as a key element of the programme. An example of a mindfulness intervention is the one developed by Alberts et al. in 2012, a programme with five core components ${ }^{(25)}$ :

(1) Mindful eating;

(2) Awareness of physical sensations;

(3) Awareness of thoughts and feelings related to eating;

(4) Acceptance and non-judgement of sensations, thoughts, feelings, and body;

(5) Awareness and step-by-step change of daily patterns and eating habits.

In contrast, many of the interventions in normal-weight populations were single-day laboratory-based studies such as completion of food tasks, rating of pleasure in food or observed food intake after a test meal. Studies often looked only at a specific practice of mindfulness such as the body scan, or mindful eating of a raisin and were therefore lower-intensity interventions than those in obese/overweight individuals.

\section{Summary of the tools/methods used to measure outcomes in intervention studies}

The following two tools were the most frequently used to measure individuals' mindfulness state and awareness:

(1) The Five Facet Mindfulness Questionnaire contained thirtynine items ${ }^{(27)}$ It: (a) measures trait (rather than state) mindfulness across five subscales (observing, describing, acting with awareness, non-judging of inner experience and non-reactivity to inner experience); and (b) reports internal consistency of 0.87 (as measured by Cronbach's $\alpha$ ).

(2) The Mindfulness Awareness Attention Scale contained fifteen items ${ }^{(62)}$. It: (a) measures trait or dispositional levels 
of mindfulness; (b) measures each item on a six-point Likert scale and measures levels of day-to-day awareness, attention and observation; (c) reports reliability of 0.82 (as measured by Cronbach's $\alpha$ ).

The Mindful Eating Questionnaire contained twenty-eight items ${ }^{(63)}$ and was the only tool used to measure mindful eating. It: (a) assesses five domains of mindful eating (disinhibition, awareness, external cues, emotional response and distraction) on a four-point Likert scale; and (b) reports reliability of $0.7-0.9$ (as measured by Cronbach's $\alpha$ ).

Intuitive eating, when measured, was evaluated using the Intuitive Eating Scale ${ }^{(64)}$ which contains twenty-one items. It: (a) assesses each item on a five-point Likert scale; (b) measures readiness to eat in response to internal cues to determine choice and quantity of food; eating to satisfy physical rather than emotional reasons; awareness and trust of internal cues; and (c) reports internal consistency of $>0.70$ (as measured by Cronbach's $\alpha$ ) and test-retest reliability of 0.90 (as measured by Cronbach's $\alpha$ ).

\section{Tools used to measure eating-related behaviours}

Eating behaviours were most frequently assessed using the Dutch Eating Behaviour Questionnaire ${ }^{(65)}$, the Eating Attitudes Test $^{(66)}$ and the Eating Disorders Examination Questionnaire ${ }^{(67)}$.

The studies reviewed used thirty-two measures of eating behaviour assessing a broad range of eating behaviours, attitudes, cognitions about food and related issues. Some of these were previously validated tools, others adapted, and others developed specifically for a study.

\section{Tools used to measure issues related to food consumption and hunger}

The majority of self-report measures involved $24 \mathrm{~h}$ recalls which were translated into energy intakes by the investigator. The studies applying objective measures involved the investigator weighing the amount of food consumed, usually within a controlled laboratory environment. The assessment of hunger was always via self-report rating scale, either on an analogue or Likert scale. There were eleven measures of the quantity of food consumed via self-report and objectively weighed.

\section{Summary of results from intervention studies}

The results of the intervention studies are presented with results in overweight/obese populations being detailed before those in normal-weight populations. Most intervention effect was seen in binge eating, emotional eating and external eating and more emphasis is given to these interventions, alongside the weightmanagement interventions in overweight/obese populations. Overall, the studies in overweight/obese populations were relatively longer and more intense compared with those carried out in normal-weight populations. Many of the observational studies were short, day-long laboratory experiments, and these are briefly summarised after the intervention studies.

A summary of the results from the intervention studies is presented in Table 2. In five of the papers in normal-weight populations, multiple related studies were reported in a single paper and the results have been collated in Table $2^{(34,53,55,58,59)}$.

Ten outcomes were given a percentage score as described previously. In studies that measured weight, binge eating, emotional eating, food intake (in overweight and obese populations) and food choice (in normal-weight populations) the score was $50 \%$ or above, indicating that most of the results were positive or neutral/mixed. Only two studies had negative results related to weight gain ${ }^{(54)}$ and an increase of energy intake ${ }^{(18)}$.

\section{Results of physiological health outcomes}

Outcomes of weight management interventions. Weight loss was seen in eight ${ }^{(13,16,28,30,31,37,40,43)}$ of the sixteen studies in overweight/obese population studies. However, the effect sizes varied widely with only three studies reporting significant weight $\operatorname{loss}^{(13,16,37)}$; the latter of these reported a mean weight loss of 12 (sE 4 ) kg over 6 months. In two studies the weight loss was minimal $^{(28,30)}$, and in two studies weight was stabilised or weight gain was less in the intervention group ${ }^{(31,43)}$. In one study results were not reported ${ }^{(40)}$.

The duration of the eight studies in overweight/obese populations that resulted in weight loss varied from 6 to 24 weeks and sample sizes varied from ten ${ }^{(13)}$ to $300^{(40)}$.

Weight-loss interventions. Of the eight studies that reported weight loss in overweight/obese populations, five of them were comprehensive mindfulness programmes ${ }^{(13,16,30,31,40)}$ where mindful eating was a component of the programme, although details of the intervention were not reported in one of these studies $^{(40)}$. The rest of these programmes taught the core elements of mindfulness such as being present in the moment and self-acceptance of the current state. The study with the greatest weight loss held group sessions for 24 weeks based on an acceptance-based behavioural intervention for weight loss; this was a pilot study, which taught key components of this approach including values, acceptance and cognitive defusion using mindfulness ${ }^{(37)}$.

Mindful eating in a restaurant setting was the sole focus of one of the eight studies ${ }^{(43)}$; the other two studies focused on the regulation of cues ${ }^{(28)}$ and used an ACT approach where mindfulness was a core component ${ }^{(37)}$.

There was some variation between interventions but the following were key elements of the mindful eating components:

(1) Mindful eating was taught by a specific exercise such as the 'mindful raisin' exercise;

(2) Mindfully attending to the eating experience by noticing smell, texture and taste of the food;

(3) Reducing the speed of eating;

(4) Guided meditations that focused on awareness of hunger and satiety;

(5) Mediations to identify eating triggers including emotional triggers;

(6) Mediations to manage cravings.

An example of how these elements were delivered in a mindfulness intervention is given in Table 3 from a nine-session MB-EAT programme for binge eating ${ }^{(16)}$. 
Table 2. Summary of coded results from intervention studies using mindfulness, mindful eating and intuitive eating techniques

\begin{tabular}{|c|c|c|c|}
\hline Health outcome & Studies ( $n$ ) (and population) & Coded results* & Score as $\%^{*} \dagger$ \\
\hline \multicolumn{4}{|l|}{ Physiological } \\
\hline \multirow[t]{4}{*}{ Weight } & 16 (overweight/obese) & 8 positive ${ }^{(13,16,28,30,31,37,40,43)}$ & 50 \\
\hline & & 8 neutral/mixed $(14,24,26,29,34,35,41,42)$ & \\
\hline & 3 (normal weight) & 2 positive $^{(34,55)}$ & 33 \\
\hline & & 1 negative ${ }^{(54)}$ & \\
\hline Physiological risk factorsł & 2 (overweight/obese) & 2 positive ${ }^{(13,26)}$ & \\
\hline Glycaemic control & 1 (overweight/obese diabetics) & 1 neutral $/$ mixed $^{(14)}$ & \\
\hline \multicolumn{4}{|l|}{ Eating behaviours } \\
\hline \multirow[t]{2}{*}{ Binge eating } & 9 (overweight/obese) & 9 positive ${ }^{(11,13,16,27,29,33,35,39,42)}$ & 100 \\
\hline & 4 (normal weight) & 4 positive $^{(45,49,60,61)}$ & 100 \\
\hline \multirow[t]{3}{*}{ Emotional eating } & 10 (overweight/obese) & 7 positive ${ }^{(11,25,30,31,33,35,39)}$ & 70 \\
\hline & & 3 neutral/mixed $(29,42,43)$ & \\
\hline & 1 (normal weight) & 1 neutral $/$ mixed $^{(54)}$ & \\
\hline \multirow[t]{3}{*}{ External eating } & 5 (overweight/obese) & 4 positive $^{(25,28,31,38)}$ & 80 \\
\hline & & 1 neutral $/ \mathrm{mixed}^{(42)}$ & \\
\hline & 1 (normal weight) & 1 positive $^{(61)}$ & \\
\hline \multirow[t]{2}{*}{ Cravings } & 1 (overweight/obese) & 1 positive ${ }^{(24)}$ & \\
\hline & 3 (normal weight) & 3 neutral/mixed $(46,56,58)$ & 0 \\
\hline Eating attitudes & 1 (normal weight) & 1 positive ${ }^{(47)}$ & \\
\hline \multirow[t]{2}{*}{ Hunger awareness } & 2 (overweight/obese) & 2 positive $^{(11,28)}$ & \\
\hline & 1 (normal weight) & 1 positive ${ }^{(48)}$ & \\
\hline Body dissatisfaction & 2 (overweight/obese) & 2 positive ${ }^{(38,39)}$ & \\
\hline \multicolumn{4}{|l|}{ Food related } \\
\hline \multirow[t]{5}{*}{ Food intake } & 4 (overweight/obese) & 3 positive ${ }^{(14,28,43)}$ & 75 \\
\hline & & 1 neutral/mixed ${ }^{(29)}$ & \\
\hline & 9 (normal weight) & 4 positive $^{(48,52,53,55)}$ & 33 \\
\hline & & 3 neutral/mixed ${ }^{(44,51,54,57)}$ & \\
\hline & & 2 negative ${ }^{(18)}$ & \\
\hline \multirow[t]{3}{*}{ Food choice } & 1 (overweight/obese) & 1 positive ${ }^{(41)}$ & \\
\hline & 5 (normal weight) & 3 positive $(15,53,55)$ & 60 \\
\hline & & 2 neutral $/$ mixed $^{(50,59)}$ & \\
\hline \multicolumn{4}{|c|}{$\begin{array}{l}\text { * Note that effect size is not accounted for in the coding of positive, negative and neutral and the code only provides an indicator of the direction of } \\
\text { results; it is not trying to assess overall evidence. The 'score' in the final column is merely a convenient summary tool in situations where there } \\
\text { have been three or more studies. }\end{array}$} \\
\hline \multicolumn{4}{|c|}{$\begin{array}{l}\text { † Calculation of score. Only health outcomes with three or more studies are 'scored': a positive study scores }+1 \text { point; a negative study scores }-1 \\
\text { point; a neutral study scores } 0 \text { points; a study with mixed results scores } 0 \text { points. The points are summed, divided by the number of studies and } \\
\text { expressed as a percentage. An example for effect of mindful eating on weight in overweight/obese: } 8 \times 1=8 / 16=0.5 \text {, giving a score }=50 \% \text {. } \\
\ddagger \text { Cholesterol and blood pressure. }\end{array}$} \\
\hline
\end{tabular}

Table 3. Example of how elements were delivered in a mindfulness intervention from a nine-session Mindfulness-Based Eating Awareness Training (MB-EAT) programme for binge eating ${ }^{(16)}$

Session

number Session content

Session 1 Introduction to self-regulation model, raisin exercise, introduction to mindfulness meditation with practice in group

Session $2 \quad$ Brief meditation (continues all sessions), mindful eating; exercise (cheese and crackers); concept of mindful eating, body scan

Session 3 Theme: Binge triggers - binge trigger meditation, mindful eating exercise (sweet, high-fat food, such as brownies)

Session $4 \quad$ Theme: Hunger cues - physiological $v$. emotional, hunger meditation, eating exercise, mindful food choices (cookies $v$. chips), healing, self-touch

Session 5 Theme: Taste satiety cues - type and level of cues, taste satiety meditation, seated yoga

Session $6 \quad$ Theme: Fullness cues - type and level of cues, fullness meditation, pot-luck meal

Session 7 Theme: Forgiveness - forgiveness meditation

Session 8 Theme: Inner wisdom - wisdom meditation, walking meditation

Session 9 Theme: Have others noticed? Where do you go from here?

The studies that did not report weight loss or where there was no intervention effect on weight were more variable in terms of duration and intensity than the studies above. A couple of the studies had a mindfulness component as part of an education programme, with an $\mathrm{ACT}^{(42)}$ and MB-EAT ${ }^{(36)}$ basis and were similar to the studies where weight loss was observed. Other studies had a narrower focus, for example internal cues ${ }^{(26)}$, mindful eating ${ }^{(29)}$ and mindful breathing ${ }^{(41)}$, and tended to have shorter duration of 4-8 weeks.

Weight loss in normal-weight populations. In two of the three normal population studies that measured weight ${ }^{(34,55)}$, weight loss was observed although effect sizes were small; these were short-term experimental studies. In one study 
undertaken among veterans with chronic conditions, weight increased $^{(54)}$

Other objective physiological measures. Three studies measured other objective physiological measures - with both improvement and mixed results reported for cardiovascular risk factors in overweight/obese populations ${ }^{(13,26)}$, and improvement in glycaemic control in diabetics ${ }^{(14,36)}$. This suggests that mindfulness interventions can create changes in physiological markers, most likely because of mindfulness-induced changes to eating habits rather than mindfulness per se having a direct effect on the physiological marker. This is an area of limited research.

\section{Eating behaviours and food-related outcomes}

A range of eating behaviours that may be associated with weight status was measured across the intervention studies including binge eating, emotional eating, external eating and cravings. Food intake and choices were also measured in some studies. A number of the studies showed that mindfulness enabled participants to become more aware of their internal states of hunger and satiety alongside the recognition of specific cues. Other studies showed that mindfulness generated more overall internal awareness, for example thoughts and feelings (which could include satiety and hunger). More generic mindfulness techniques emerged strongly in the studies. These included the body scan exercise and mindful breathing exercises. Acceptance-based exercises were used in a number of studies connected to recognition of emotions and thoughts.

\section{Eating-related behaviours}

Binge eating. Binge eating symptoms were measured in nine studies in overweight/obese populations. All the studies reported positive changes in binge eating symptoms over the course of the study, as evidenced by self-report measures. In four of the studies binge eating was a specific target of the intervention ${ }^{(11,16,33,39)}$. The first of these studies was the initial exploratory work with mindfulness and any eating-related behaviour ${ }^{(11)}$.

Binge eating symptoms were assessed in four studies in normal-weight populations ${ }^{(45,49,60,61)}$; three were undertaken in binge-eating-disorder populations. Positive results were seen in all studies.

Collectively, these results suggest strong evidence for the efficacy of mindfulness in reducing the frequency and intensity of binge eating symptoms. This is particularly notable given the high degree of heterogeneity between the studies in terms of design and intervention.

Binge eating interventions. Specific mindful eating strategies were included in the majority of studies that measured binge eating. In many cases, this was complemented with generic mindfulness meditations such as breathing techniques and body scans often as part of a mindfulness-based curriculum, for example, MB-EAT ${ }^{(16)}$, MBSR $^{(39,60)}$ or mindfulness-based cognitive therapy ${ }^{(27,33)}$. Mindfulness was also sometimes combined with $\mathrm{ACT}^{(42,49)}$ or action-based cognitive therapy ${ }^{(45)}$. Awareness of internal hunger cues was not as prevalent in these studies. These findings suggest that in the management of binge eating, the ability to eat mindfully is a key technique.

Emotional eating. Ten studies measured emotional eating in overweight/obese populations. Reductions in emotional eating were reported in seven of these studies $^{(11,25,30,31,33,35,39)}$ and three studies showed no change ${ }^{(29,42,43)}$. One of the studies where there was no effect was undertaken among children ${ }^{(29)}$, another was focused on restaurant eating only ${ }^{(43)}$, and the third was an ACT-based intervention where mindfulness was not the sole focus ${ }^{(42)}$. These positive results provide support for the use of mindfulness-based interventions in reducing emotional eating in overweight or obese populations.

Only one study measured emotional eating in a normal-weight population $^{(54)}$ where no impact of the MBSR intervention was seen; this population of veterans with chronic conditions may not be generalisable.

External eating. External eating was measured in five of the studies within an overweight/obese population. Four of these studies showed a positive change in external eating and were undertaken in populations suffering from stress eating, problematic eating, disordered eating attitudes, and children learning specifically about cues ${ }^{(25,28,31,38)}$. One study, in this population reported a neutral result ${ }^{(42)}$. This was an ACT intervention study over 6 months among women. External eating was measured in one study in a normal-weight population suffering from binge eating and the intervention reduced binge eating episodes.

Taken together, these findings suggest that mindfulness can be helpful in reducing external eating and reduce the responsiveness of individuals to external cues such as food packaging and time of day. The evidence in normal-weight populations appears to be limited.

External eating and emotional eating interventions. All studies that looked at external and emotional eating used specific mindful eating techniques in their interventions. Mindfulness techniques such as body scans, breathing exercises and awareness of bodily sensation were utilised in the studies to a varying extent. In the studies done in overweight and obese populations the mindful practice was part of a wider curriculum $^{(11,25,30,31)}$, or sometimes supported by a one-day workshop $^{(38)}$. This suggests that in order to effect change in external and emotional eating, it is important to use techniques that increase awareness of internal states, combined with acceptance techniques. Combining these with mindful eating seems to be important.

Cravings. Cravings were measured in one study in an overweight/obese population ${ }^{(24)}$, which showed a positive response to a mindfulness-based intervention, and in three studies in normal-weight populations, all of which showed mixed/neutral results ${ }^{(46,56,58)}$. These studies were all laboratory-based studies and two of them were day-long studies only. Given the limited 
number of studies and their short duration, the role of mindfulness in reducing cravings remains to be fully elucidated.

Hunger awareness. Hunger awareness was measured in two overweight/obese populations and positive results were reported in both studies ${ }^{(11,28)}$. In one of these studies a 12-week curriculum was developed which focused on 'Regulation of Cues'; children in this group had significant reductions in eating in response to external cues and negative states, and attending to food responsiveness ${ }^{(28)}$. A study in normal-weight students which made one focus group concentrate on the sensory experience of foods showed a lower pre-snack hunger rating than either of the control groups ${ }^{(48)}$. These results suggest that mindfulness is effective in increasing hunger awareness, although this was only measured in three studies, so the finding should be considered preliminary.

Body dissatisfaction. Two studies measured body dissatisfaction in relation to mindfulness interventions in overweight/ obese populations ${ }^{(38,39)}$. Both of these studies demonstrated positive results, but this is a limited evidence base from which to suggest that mindfulness can support improvements in body dissatisfaction.

\section{Food intake and choice}

Food intake was measured in four studies in overweight/obese populations and three of these reported a reduced energy intake, as measured by self-report via $24 \mathrm{~h}$ recalls or $\mathrm{FFQ}^{(28,36,43)}$. The first two of these studies were comprehensive education programmes while the third focused on intake at lunchtime only. One study did not find a reduction in energy intake as a result of their intervention among children and their parents ${ }^{(29)}$.

Food intake was measured in nine studies in normal-weight populations, with four studies reporting a reduction of food intake $^{(48,52,53,55)}$, four reporting neutral results ${ }^{(44,51,54,57)}$ and one reporting negative results ${ }^{(18)}$. One of these studies reported that higher levels of intuitive eating was associated with increased food intake in a permissive environment which was an unexpected finding ${ }^{(18)}$. No change in food intake was seen in a study among veterans with chronic health problems ${ }^{(54)}$. In contrast to the studies in overweight/obese populations, in all but one study ${ }^{(54)}$, food intake was measured in short-duration, low-intensity experiments in laboratory settings using test meals or snacks rather than in a free-living situation.

These results suggest that more evidence is needed before it is possible to say if mindfulness and/or mindful eating can reduce the quantity of food consumed.

Food choice was measured in one study in overweight/obese populations which looked at lunches purchased and showed positive trend in choosing healthier meals ${ }^{(41)}$. Food was measured in five studies in normal-weight populations. Three of these studies reported positive food choices ${ }^{(15,53,55)}$ as a result of mindfulness intervention and two studies reported mixed/neutral results ${ }^{(50,59)}$. As per food intake, these were lowintensity, laboratory studies asking participants to make a snack choice, with the healthier choice being determined as positive.
Results - observational studies

\section{Theoretical basis}

Eight papers focused on intuitive eating ${ }^{(18,68-75)}$, five papers focused on mindfulness ${ }^{(76-80)}$, two papers focused on mindful eating $^{(81,82)}$ and one paper had a combined mindfulness and mindful eating approach ${ }^{(83)}$.

\section{Summary of results from observational studies}

Sixteen observational studies were identified in the literature search and a summary of the associations observed are shown in the Table 4; it should be noted that some studies investigated more than one association. Many of the observational studies looked at associations between an aspect of eating or mindfulness and related it to an outcome in an existing cohort, for example level of mindfulness, or intuitive eating and BMI. Associations were made using the tools and scales already outlined in the intervention studies.

Two of the cohorts were substantial, with $1000^{(72)}$ and $2000^{(69)}$ subjects, respectively. The cohort populations were diverse, with six undertaken among university students ${ }^{(69-71,73,80,82)}$ and two taken among other young adult populations ${ }^{(68,71)}$. The remaining cohorts were spread across a wide age spectrum from middle school $^{(72)}$ to older obese adults ${ }^{(78)}$. Four of the cohorts were from overweight/obese populations of which two were waiting for bariatric surgery ${ }^{(76,77)}$.

Intuitive eating was associated with a lower BMI in four studies $^{(68,70,74,75)}$. Other observations included a decreased emotional eating with higher levels of mindfulness ${ }^{(76,77,79)}$ and a higher level of everyday mindfulness was related negatively to self-report serve size of energy-dense food ${ }^{(83)}$. In one of

Table 4. Summary of coded results from observational studies showing the association between mindfulness, mindful eating and intuitive eating and parameters related to health measures and eating behaviours

\begin{tabular}{|c|c|c|}
\hline Associations & $\begin{array}{l}\text { Studies } \\
\qquad(n)\end{array}$ & Coded result* \\
\hline Intuitive eating and decreased BMI & 4 & Positive ${ }^{(68,70,74,75)}$ \\
\hline Mindful eating and decreased BMI & 1 & Positive ${ }^{(82)}$ \\
\hline $\begin{array}{l}\text { Intuitive eating and blood markers of } \\
\text { cardiovascular risk }\end{array}$ & 1 & Positive ${ }^{(70)}$ \\
\hline Mindfulness and decreased portion size & 1 & Positive ${ }^{(83)}$ \\
\hline Intuitive eating and observing hunger cues & 1 & Positive ${ }^{(72)}$ \\
\hline Mindfulness and decreased binge eating & 2 & Positive ${ }^{(76,80)}$ \\
\hline Mindfulness and decreased emotional eating & 3 & Positive ${ }^{(76,77,79)}$ \\
\hline Mindfulness and external eating & 1 & Positive ${ }^{(77)}$ \\
\hline Mindfulness and reduced cravings & 1 & Positive ${ }^{(78)}$ \\
\hline Mindfulness and restrained eating & 1 & Positive ${ }^{(75)}$ \\
\hline Intuitive eating and freedom to eat & 1 & Positive ${ }^{(73)}$ \\
\hline Intuitive eating and interoceptive sensitivity & 1 & Positive ${ }^{(71)}$ \\
\hline $\begin{array}{l}\text { Trait mindfulness and returning baseline } \\
\text { brain network post-food cue exposure }\end{array}$ & 1 & Positive ${ }^{(78)}$ \\
\hline Mindful eating and mental well-being & 1 & Positive ${ }^{(81)}$ \\
\hline $\begin{array}{l}\text { Intuitive eating and more pleasure } \\
\text { associated with food }\end{array}$ & 1 & Positive ${ }^{(75)}$ \\
\hline $\begin{array}{l}\text { Intuitive eating and increased external and } \\
\text { introjection regulation in behaviour } \\
\text { questionnaire }\end{array}$ & 1 & Positive ${ }^{(69)}$ \\
\hline
\end{tabular}

* Note that effect size is not accounted for in the coding of positive. The code only provides an indicator of direction of results and is not trying to assess overall evidence. 
the large cohorts in young adults, there was no difference in intuitive eating by age or sex ${ }^{(68)}$ while another study which compared males and females in a young adult population found higher levels of anti-dieting and self-care subscales of the intuitive eating scale ${ }^{(69)}$. One study found males trusting their bodies more than females on when to eat ${ }^{(68)}$ but this was reversed in a second study ${ }^{(72)}$. Another study looking at associations with intuitive eating showed that those who consistently overrode fullness feelings were more likely to use moderate to extreme effort to maintain their weight ${ }^{(73)}$.

Interoceptive sensitivity is a measure of awareness of the subject's bodily state; this was positively related to higher levels of intuitive eating ${ }^{(71)}$. An online survey in Pakistan showed a significantly positive relationship between overall mindful eating and mental well-being ${ }^{(81)}$. In one of the group of bariatric patients, greater mindfulness, particularly awareness, was related to fewer binges and emotional eating $^{(76)}$. Another observation in bariatric patients showed that mindfulness was positively associated with restrained eating behaviour $^{(77)}$.

These studies indicate that both mindfulness and intuitive eating showed promise in positively influencing complex relationships with food and eating behaviours.

\section{Potential associated mechanisms}

The literature search showed a growing evidence base for mindfulness, mindful eating and intuitive eating, but the papers retrieved generally did not discuss underlying mechanisms. Therefore, a further search of the scientific literature was undertaken to provide potential explanations for the observed effects. We identified three separate hypothesised mechanisms that were suggested by functional MRI (fMRI) results; fMRI measures brain activity by detecting associated changes in blood flow.

\section{Increased awareness of, and increased responsiveness to,} internal physical cues

Interoception can be measured by an individual's ability to report their heart rate. Interoception is connected with the mindful awareness of an individual's internal state, especially in relation to physical factors such as hunger and satiety. Increased awareness enables individuals to eat in response to these physical cues of hunger and satiety.

Mindful eating has been shown to slow down consumption of a meal and allows both registration of feelings of fullness and greater control over eating ${ }^{(84,85)}$. Additionally, a slower pace of eating reduces overall energy consumption as individuals feel full on a smaller quantity of food ${ }^{(86)}$.

One study using fMRI showed a decrease in mid-line cortical structures associated with interoception during a mindfulness body scan. This suggests an increased awareness of internal state $^{(87)}$. In another study, fMRI results showed reduced activation in the insula ${ }^{(88)}$ which is the part of the cerebral cortex implicated in self-awareness and perception and hence is linked with interoceptive awareness.
Increased awareness of, and reduced responsiveness to, internal emotional cues

Mindfulness trains individuals to notice distressing thoughts, emotions and sensations. By increasing awareness of these cues, individuals are then able to tolerate their distress and not allow these non-physical cues make them eat more ${ }^{(77)}$.

By learning to bring the experience fully into awareness many types of distress that would have provoked an automatic reaction can be tolerated. Thus, distress tolerance is increased and automaticity reduced. In relation to emotional eating, mindfulness reduces misperception of emotions as hunger and therefore stops increased eating in response to non-physical cues ${ }^{(77)}$.

As defusion-reduced reactivity enhances tolerance to nonphysical emotional cues, the cultivation of mindfulness becomes self-reinforcing ${ }^{(89)}$. Further, mindfulness increases the capability to relax when faced with negative events and emotion. This has been found to be especially true in reducing impulsivity, which is associated with mindless overeating ${ }^{(77)}$.

fMRI studies have shown altered activity levels in the amygdala related to general emotional arousal/salience following mindfulness training. Decreased activation has been found in the amygdala, the parahippocampal area and the insula during perception of negative stimuli in subjects but these activity levels have been regulated following mindfulness training ${ }^{(90)}$. Others have used fMRI to show that mindfulness makes an impact on the neural systems underlying emotional regulation. It appears to regulate amygdala functioning via functional connectivity from the medial prefrontal cortex ${ }^{(91)}$.

fMRI studies conducted on individuals practising mindfulness have found a significant reduction in activity in the anterior cingulated cortex region which plays a role in the processing of emotion. 'Findings collectively show that mindfulness meditation has an overall "quietening" effect on brain regions associated with subjective and cognitive appraisal of emotions ${ }^{(87)}$. Significant selfreported differences in awareness of emotion are unlikely to be simply due to a blunting of the emotional experience but 'represent more fundamental change in regulatory response, ${ }^{\text {,(88) }}$.

Anatomical studies lend further support to changes in the areas of the brain associated with emotional regulation. Studies conducted pre- and post-MRI on participants following an 8 -week mindfulness programme found an increase in grey matter concentration in the left hippocampus and posterior cingulated cortex, temporo-parietal junction, and cerebellum areas associated with learning and memory processes, emotional regulation and self-referential processing ${ }^{(92)}$.

Increased awareness of, and reduced responsiveness to, external cues

Overeating may reflect a non-mindful reliance on environmental or external cues (for example, packaging size), so that the cues destroy the subject's ability to self-monitor the food they eat ${ }^{(83)}$. The use of mindfulness is designed to interrupt these automatic, non-conscious external influences ${ }^{(93)}$. Increasing awareness of an individual's reactions to attractive food items, and a recognition of these reactions as passing mental states rather than real experiences, reduced the likelihood of individuals initiating 
purchasing and eating behaviours ${ }^{(59)}$. Mindfulness practice ${ }^{(83)}$ and mindful eating specifically reduced the impact of the external norms around consumption and enabled individuals to reduce their food intake ${ }^{(34,94)}$.

fMRI has been used to compare results of subjects who received an 8-week mindfulness-based stress reduction course with those of a control group ${ }^{(95)}$. When asked to pay attention to the external sounds in the scanner environment, the mindfulness group showed increased functional connectivity in auditory and visual networks and the areas associated with attentional and self-referential processes.

These observations from fMRI studies are of interest but are insufficient on their own to explain the mechanism by which mindfulness exerts its effects. More research is needed to fully elucidate further mechanisms and these must explain other influencing behavioural factors.

\section{Discussion}

From the papers reviewed there appears to be reasonable evidence that mindfulness, including mindful eating techniques, can help to change eating behaviours. The evidence for the effectiveness of mindfulness and mindful eating in binge eating disorders appears to be the strongest with consistently positive results in both overweight/obese populations and those of normal-weight status. There was also a positive effect on emotional eating as a result of mindfulness interventions and in a lesser number of studies a positive result was seen for reducing eating in response to external cues. This is consistent with the findings of O'Reilly et al. in $2014^{(23)}$, who examined the role of mindfulness with obesity-related behaviours and a review looking at the effectiveness of mindfulness in binge eating ${ }^{(19,23)}$.

Food cravings, eating attitudes and body dissatisfaction were explored in only a limited number of studies. Although binge eating and emotional eating are important obesity-related behaviours, the results for the use of mindfulness in achieving weight loss in overweight/obese individuals were mixed and generally effect sizes were small. This is not surprising given the complex and multifaceted nature of obesity, which requires a multi-pronged treatment approach. The diversity of the interventions makes it difficult to identify the effective elements. With regard to the interventions that did not report weight loss there were no striking differences from the ones that were successful, except that a couple of the studies were of lower intensity and shorter duration. This diverse range of treatments included mindfulness as part of a third-wave therapy such as ACT, mindfulness curricula based on established programmes in other conditions adapted for eating, for example MBSR and mindfulness-based cognitive therapy, through to less intense interventions which employed mindfulness techniques as stand-alone interventions. However, the majority of successful interventions included mindful eating as part of a comprehensive mindfulness programme. These mindfulness programmes contained core elements of mindfulness including meditation, awareness and being present. A recent review on the effectiveness of mindfulness in achieving weight loss found that the majority of studies did result in weight loss but the extent to which mindfulness could be attributed to the effect was not apparent ${ }^{(22)}$. This study also noted the wide variation in interventions and methodological weaknesses.

It is important to recognise that many of the studies in binge eating and weight loss did not have a non-intervention control group and did not measure changes in mindfulness. Furthermore, few of the studies had a follow-up so it is not possible to determine long-term success. Despite these methodological weaknesses, the use of mindfulness and mindful eating in weight management and obesity-related behaviours shows promise and the field warrants further investigation.

In contrast to the intervention studies in overweight/obese studies, nearly half of the studies in normal-weight populations were laboratory-based, day-long studies, many of which were carried out among students. A small number of the studies were mediumlength interventions (i.e. 6-16 weeks' duration), which highlights the great variation in intensity of factors in the interventions in normal-weight subjects. The interventions were diverse and included teaching intuitive eating, providing mindfulness information, a mindfulness group intervention for 4 months, body scanguided imagery, mindful eating exercises and mindful awareness. Furthermore, the outcomes varied widely, so comparison between studies was not possible. The outcomes included food choices, food intake, and the following eating behaviours: cravings, binge eating, internal cues, and the relationship with food. Overall, the results indicated a positive role for mindfulness and/or mindful eating on influencing eating behaviours. This avenue of research also merits further research as this has the potential to be relevant to public health nutrition messaging regarding food choices.

The observational studies also provide direction for future research but, by their nature, are evidence of association only. It is interesting to note that, in these observational studies, the focus was on intuitive eating whereas only two interventions ${ }^{(26,47)}$ were based on intuitive eating. This indicates that intuitive eating is an emerging topic and further research is needed to examine its potential in positively having an impact on eating behaviours. The findings of the observational studies were unanimously positive, demonstrating a beneficial association of mindfulness/mindful eating or intuitive eating.

Overall, many studies were limited by small subject numbers and were also predominately carried out among female populations, the majority of whom were from Caucasian backgrounds. Given the importance of weight reduction in the management of type 2 diabetes and in CVD, it was surprising not to find more studies in these patient groups. This is likely to be an area of study in the future.

While research into the mechanisms of action of mindfulness is ongoing, particularly in relation to mindful eating, concomitant psychological and physiological findings have yielded three key hypotheses about the associated processes involved in the brain. Results from fMRI studies show that mindfulness is associated with reduced amygdala activity and may reduce emotional arousal. Mindfulness possibly works by increasing inner physical cues to eat (hunger and satiety), and decreasing the emotional and external cues to eat.

Only two studies reported negative results regarding the mindfulness or mindful eating intervention (small increases in weight and food intake), and one of these was in a population that is not generalisable. 


\section{Strengths and limitations of the present structured review}

This structured review provides a literature review about the potential of mindfulness, mindful eating, and intuitive eating to make an impact on eating behaviours. It takes a broad approach, which provides perspective on the state of current research and direction of future research. It is common in research for observational studies to precede interventions where observed associations can be tested in experimental conditions. The description of the interventions and the tools used for outcome measures adds a new element to the systematic reviews undertaken to date in this area.

The review was never intended to be a systematic review and, as such, cannot make categorical statements on the quality of the evidence base. Furthermore, a rigorous critical appraisal of the literature is beyond the scope of this review. 'Attention' was not included in the search terms for the interventions undertaken and it has since become apparent that this would have been a relevant search term. Attentive eating is a similar concept to mindful eating and has been shown to reduce food intake ${ }^{(96,97)}$.

\section{Conclusions}

Mindfulness and mindful eating interventions appear to be most successful in the reduction of binge eating regardless of weight status. Mindfulness and mindful eating interventions also reduced emotional eating and eating in response to external cues which are important behaviours related to obesity.

In overweight individuals, the impact of mindfulness and mindful eating on reducing body weight was mixed; it might be that mindfulness and mindful eating have a role to play in the prevention of weight gain. Mindfulness/mindful eating resulted in a positive shift in food habits and in the quantities of food consumed in some studies. There is evidence both for the efficacy of one-off mindfulness interventions and also for the benefit of repeated practice on improving outcomes. On the basis of this review, encouraging a mindful approach to eating appears to be a positive message to be included in general weight-management advice to the public.

\section{Acknowledgements}

The present review was funded by an unconditional grant from Mondelez International. The authors are all independent freelance consultants and followed guidelines for scientific integrity from the inception of the review to final drafting of the manuscript. Mondelez International did not have any role in undertaking the review or interpreting the results.

The authors have no declarations of interest to declare.

\section{References}

1. National Obesity Observatory \& Public Health England (2011) Adult obesity. http://www.noo.org.uk/NOO_about_obesity/ adult_obesity (accessed January 2017).

2. Food Standards Agency (2016) Our food future. https://www. food.gov.uk/news-updates/campaigns/ourfoodfuture (accessed January 2017).

3. Bishop SR, Lau M, Shapiro S, et al. (2004) Mindfulness: a proposed operational definition. Clin Psych Sci Pract 11, 230-241.
4. Kabat-Zinn J (1990) Full Catastrophe Living: Using the Wisdom of Your Body and Mind to Face Stress, Pain, and Illness. New York: Delacorte Press.

5. Chiesa A \& Serretti A (2011) Mindfulness-based interventions for chronic pain: a systematic review of the evidence. $J$ Altern Complement Med 17, 83-93.

6. Zeidan F, Grant JA, Brown CA, et al. (2012) Mindfulness meditation-related pain relief: evidence for unique brain mechanisms in the regulation of pain. Neurosci Lett 520, 165-173.

7. Klainin-Yobas P, Cho MA \& Creedy D (2012) Efficacy of mindfulness-based interventions on depressive symptoms among people with mental disorders: a meta-analysis. Int $J$ Nurs Stud 49, 109-121.

8. Piet J \& Hougaard E (2011) The effect of mindfulness-based cognitive therapy for prevention of relapse in recurrent major depressive disorder: a systematic review and meta-analysis. Clin Psych Rev 31, 1032-1040.

9. Chiesa A \& Serretti A (2009) Mindfulness-based stress reduction for stress management in healthy people: a review and meta-analysis. J Altern Complement Med 15, 593-600.

10. Hofmann SG, Grossman P \& Hinton DE (2011) Lovingkindness and compassion meditation: potential for psychological interventions. Clin Psych Rev 31, 1126-1132.

11. Kristeller JL \& Hallett CB (1999) An exploratory study of a meditation-based intervention for binge eating disorder. $J$ Health Psychol 4, 357-363.

12. Kristeller J, Baer R \& Quilian-Wolever R (2006) Mindfulnessbased approaches to eating disorders. In Mindfulness-based Treatment Approaches, pp. 75-93 [R Baer, editor]. Burlington, MA: Academic Press.

13. Dalen J, Smith BW, Shelley BM, et al. (2010) Pilot study: Mindful Eating and Living (MEAL): weight, eating behavior, and psychological outcomes associated with a mindfulnessbased intervention for people with obesity. Complement Ther Med 18, 260-264.

14. Miller CK, Kristeller JL, Headings A, et al. (2014) Comparison of a mindful eating intervention to a diabetes self-management intervention among adults with type 2 diabetes: a randomized controlled trial. Health Educ Behav 41, 145-154.

15. Hendrickson KL \& Rasmussen EB (2013) Effects of mindful eating training on delay and probability discounting for food and money in obese and healthy-weight individuals. Behav Res Ther 51, 399-409.

16. Kristeller JL, Wolever RQ \& Sheets V (2014) Mindfulness-Based Eating Awareness Training (MB-EAT) for binge eating: a randomized clinical trial. Mindfulness $\mathbf{5}$, 282-297.

17. Mathieu J (2009) What should you know about mindful and intuitive eating? J Am Diet Assoc 109, 1982-1987.

18. Anderson DA, Schaumberg K, Anderson LM, et al. (2015) Is level of intuitive eating associated with plate size effects? Eat Behav 18, 125-130.

19. Godfrey KM, Gallo LC \& Afari N (2015) Mindfulness-based interventions for binge eating: a systematic review and metaanalysis. J Behav Med 38, 348-362.

20. Katterman SN, Kleinman BM, Hood MM, et al. (2014) Mindfulness meditation as an intervention for binge eating, emotional eating, and weight loss: a systematic review. Eat Behav 15, 197-204.

21. Godsey J (2013) The role of mindfulness based interventions in the treatment of obesity and eating disorders: an integrative review. Complement Ther Med 21, 430-439.

22. Olson KL \& Emery CF (2015) Mindfulness and weight loss: a systematic review. Psychosom Med 77, 59-67. 
23. O'Reilly GA, Cook L, Spruijt-Metz D, et al. (2014) Mindfulnessbased interventions for obesity-related eating behaviours: a literature review. Obes Rev 15, 453-461.

24. Alberts HJ, Mulkens S, Smeets M, et al. (2010) Coping with food cravings. investigating the potential of a mindfulnessbased intervention. Appetite 55, 160-163.

25. Alberts HJ, Thewissen R \& Raes L (2012) Dealing with problematic eating behaviour. The effects of a mindfulnessbased intervention on eating behaviour, food cravings, dichotomous thinking and body image concern. Appetite $\mathbf{5 8}$, 847-851.

26. Bacon L, Stern JS, Van Loan MD, et al. (2005) Size acceptance and intuitive eating improve health for obese, female chronic dieters. J Am Diet Assoc 105, 929-936.

27. Baer R, Fischer S \& Huss D (2005) Mindfulness and acceptance in the treatment of disordered eating. $J$ Rat-Emo Cognitive-Behav Ther 23, 281-300.

28. Boutelle KN, Zucker N, Peterson CB, et al. (2014) An intervention based on Schachter's externality theory for overweight children: the regulation of cues pilot. I Pediatr Psychol 39, 405-417.

29. Boutelle KN, Zucker NL, Peterson CB, et al. (2011) Two novel treatments to reduce overeating in overweight children: a randomized controlled trial. J Consult Clin Psychol 79, 59-71.

30. Corsica J, Hood MM, Katterman S, et al. (2014) Development of a novel mindfulness and cognitive behavioral intervention for stress-eating: a comparative pilot study. Eat Behav 15, 694-699.

31. Daubenmier J, Kristeller J, Hecht FM, et al. (2011) Mindfulness intervention for stress eating to reduce cortisol and abdominal fat among overweight and obese women: an exploratory randomized controlled study. J Obes 2011, 651936.

32. Kidd LI, Graor CH \& Murrock CJ (2013) A mindful eating group intervention for obese women: a mixed methods feasibility study. Arch Psychiatr Nurs 27, 211-218.

33. Leahey T, Crowther J \& Irwin S (2008) A cognitive-behavioral mindfulness group therapy intervention for the treatment of binge eating in bariatric surgery patients. Cog Behav Pract 15, 364-375.

34. Mantzios M \& Wilson JC (2014) Making concrete construals mindful: a novel approach for developing mindfulness and self-compassion to assist weight loss. Psychol Health 29, 422-441.

35. Mason AE, Lustig RH, Brown RR, et al. (2015) Acute responses to opioidergic blockade as a biomarker of hedonic eating among obese women enrolled in a mindfulness-based weight loss intervention trial. Appetite 91, 311-320.

36. Miller CK, Kristeller JL, Headings A, et al. (2012) Comparative effectiveness of a mindful eating intervention to a diabetes self-management intervention among adults with type 2 diabetes: a pilot study. J Acad Nutr Diet 112, 1835-1842.

37. Niemeier HM, Leahey T, Palm Reed K, et al. (2012) An acceptance-based behavioral intervention for weight loss: a pilot study. Behav Ther 43, 427-435.

38. Pearson A, Follette V \& Hayes S (2012) A pilot study of acceptance and commitment therapy as a workshop intervention for body dissatisfaction and disordered eating attitudes. Cog Behav Pract 19, 181-197.

39. Smith BW, Shelley BM, Leahigh L, et al. (2006) A preliminary study of the effects of a modified mindfulness intervention on binge eating. J Evidence-Base Complem Altern Med 11, 133-143.

40. Soleimaninanadegani M \& Shahmohammadi N (2013) The impact of mindfulness-based cognitive therapy on anthropometric indices balance in high-school students in Iran. Proc Social Behav Sci 84, 542-548.
41. Stites SD, Singletary SB, Menasha A, et al. (2015) Pre-ordering lunch at work. Results of the what to eat for lunch study. Appetite 84, 88-97.

42. Tapper K, Shaw C, Ilsley J, et al. (2009) Exploratory randomised controlled trial of a mindfulness-based weight loss intervention for women. Appetite 52, 396-404.

43. Timmerman GM \& Brown A (2012) The effect of a mindful restaurant eating intervention on weight management in women. J Nutr Educ Behav 44, 22-28.

44. Cavanagh K, Vartanian LR, Herman CP, et al. (2014) The effect of portion size on food intake is robust to brief education and mindfulness exercises. J Health Psychol 19, 730-739.

45. Courbasson CM, Nishikawa Y \& Shapira LB (2011) Mindfulness-action based cognitive behavioral therapy for concurrent binge eating disorder and substance use disorders. Eat Disord 19, 17-33.

46. Hamilton J, Fawson S, May J, et al. (2013) Brief guided imagery and body scanning interventions reduce food cravings. Appetite 71, 158-162.

47. Healy N, Joram E, Matvienko O, et al. (2015) Impact of an intuitive eating education program on high school students' eating attitudes. Health Educ 115, 214-228.

48. Higgs S \& Donohoe JE (2011) Focusing on food during lunch enhances lunch memory and decreases later snack intake. Appetite 57, 202-206.

49. Hill M, Masuda A, Melcher H, et al. (2015) Acceptance and commitment therapy for women diagnosed with binge eating disorder: a case-series study. Cog Behav Pract 22, 367-378.

50. Hong P, Lishner D \& Han K (2014) Mindfulness and eating: an experiment examining the effect of mindful raisin eating on the enjoyment of sampled food. Mindfulness 5, 80-87.

51. Jacobs J, Cardaciotto L, Block-Lerner J, et al. (2013) A pilot study of a single-session training to promote mindful eating. Adv Mind Body Med 27, 18-23.

52. Jenkins KT \& Tapper K (2014) Resisting chocolate temptation using a brief mindfulness strategy. Br J Health Psychol 19, 509-522.

53. Jordan C, Wanga W, Donatoni L, et al. (2014) Mindful eating: trait and state mindfulness predict healthier eating behavior. Pers Indiv Diff 68, 107-111.

54. Kearney DJ, Milton ML, Malte CA, et al. (2012) Participation in mindfulness-based stress reduction is not associated with reductions in emotional eating or uncontrolled eating. Nutr Res 32, 413-420.

55. Kidwell B, Hasford J \& Hardesty D (2015) Emotional ability training and mindful eating. J Marketing Res LII, 105-119.

56. Lacaille J, Ly J, Zacchia N, et al. (2014) The effects of three mindfulness skills on chocolate cravings. Appetite $\mathbf{7 6}$, 101-112.

57. Marchiori D \& Papies EK (2014) A brief mindfulness intervention reduces unhealthy eating when hungry, but not the portion size effect. Appetite 75, 40-45.

58. May J, Andrade J, Batey H, et al. (2010) Less food for thought. Impact of attentional instructions on intrusive thoughts about snack foods. Appetite 55, 279-287.

59. Papies E, Barsalou L \& Custers R (2012) Mindful attention prevents mindless impulses. Soc Psych Pers Sci 3, 291-299.

60. Smith BW, Shelley BM, Dalen J, et al. (2008) A pilot study comparing the effects of mindfulness-based and cognitivebehavioral stress reduction. I Altern Complem Med 14, 251-258.

61. Woolhouse H, Knowles A \& Crafti N (2012) Adding mindfulness to CBT programs for binge eating: a mixed-methods evaluation. Eat Disord 20, 321-239. 
62. Brown KW \& Ryan RM (2003) The benefits of being present: mindfulness and its role in psychological well-being. J Pers Soc Psychol 84, 822-848.

63. Framson C, Kristal AR, Schenk JM, et al. (2009) Development and validation of the Mindful Eating Questionnaire. J Am Diet Assoc 109, 1439-1444.

64. Tylka TL \& Kroon Van Diest AM (2013) The Intuitive Eating Scale-2: item refinement and psychometric evaluation with college women and men. I Couns Psychol 60, 137-153.

65. Van Strien T, Frijters JER \& Bergers GPA (1986) The Dutch Eating Behaviour Questionnaire (DEBQ) for assessment of restrained, emotional, and external eating behavior. Int J Eat Disord 5, 295-316.

66. Garner D, Olmsted MP, Bohr Y, et al. (1982) The Eating Attitudes Test - psychometric features and clinical correlates. Psychol Med 12, 871-878.

67. Black CMD \& Wilson GT (1996) Assessment of eating disorders: interview $v$. questionnaire. Int $J$ Eat Disord 20, 43-50.

68. Denny KN, Loth K, Eisenberg ME, et al. (2013) Intuitive eating in young adults. Who is doing it, and how is it related to disordered eating behaviors? Appetite 60, 13-19.

69. Gast J, Madanat H \& Nielson AC (2012) Are men more intuitive when it comes to eating and physical activity? Am J Mens Health 6, 164-171.

70. Hawks S, Madanat H, Hawks J, et al. (2005) The relationship between intuitive eating and health indicators among college women. Am J Health Educ 36, 331-316.

71. Herbert BM, Blechert J, Hautzinger M, et al. (2013) Intuitive eating is associated with interoceptive sensitivity. Effects on body mass index. Appetite 70, 22-30.

72. Moy J, Petrie TA, Dockendorff S, et al. (2013) Dieting, exercise, and intuitive eating among early adolescents. Eat Behav 14, 529-532.

73. Outland L, Madanat $H$ \& Rust F (2013) Intuitive eating for a healthy weight. Primary Health Care 23, 22-28.

74. Sairanen E, Tolvanen A, Karhunen L, et al. (2015) Psychological flexibility and mindfulness explain intuitive eating in overweight adults. Behav Modif 39, 557-579.

75. Smith $\mathrm{T} \&$ Hawks $\mathrm{S}$ (2006) Intuitive eating, diet composition, and the meaning of food in healthy weight promotion. $A m J$ Health Educ 37, 130-136.

76. Levin ME, Dalrymple K, Himes S, et al. (2014) Which facets of mindfulness are related to problematic eating among patients seeking bariatric surgery? Eat Behav 15, 298-305.

77. Ouwens MA, Schiffer AA, Visser LI, et al. (2015) Mindfulness and eating behaviour styles in morbidly obese males and females. Appetite 87, 62-67.

78. Paolini B, Burdette JH, Laurienti PJ, et al. (2012) Coping with brief periods of food restriction: mindfulness matters. Front Aging Neurosci 4, 13.
79. Pidgeon A, Lacota K \& Champion J (2013) The moderating effects of mindfulness on psychological distress and emotional eating behaviour. Aust Psychol 48, 262-269.

80. Roberts K \& Danoff-Burg S (2010) Mindfulness and health behaviors: is paying attention good for you? J Am Coll Health 59, $165-173$.

81. Khan Z \& Zadeh Z (2014) Mindful eating and its relationship with mental well-being. Soc Behav Sci 159, 69-73.

82. Moor K, Scott A \& McIntosh W (2013) Mindful eating and its relationship to body mass index and physical activity among university students. Mindfulness 4, 269-274.

83. Beshara M, Hutchinson AD \& Wilson C (2013) Does mindfulness matter? Everyday mindfulness, mindful eating and selfreported serving size of energy dense foods among a sample of South Australian adults. Appetite 67, 25-29.

84. Gerweck C \& Celentano J (2015) The real weighty issue: losing is just the beginning. Am J Life Med 9, 212-216.

85. Lofgren I (2015) Mindful eating: an emerging approach for healthy weight management. Am J Life Med 9, 212-216.

86. Monroe J (2015) Mindful eating: principles and practice. Am J Life Med 9, 217-220.

87. Ives-Deliperi VL, Solms M \& Meintjes EM (2011) The neural substrates of mindfulness: an fMRI investigation. Soc Neurosci 6, 231-242.

88. Farb NA, Anderson AK, Mayberg H, et al. (2010) Minding one's emotions: mindfulness training alters the neural expression of sadness. Emotion 10, 25-33.

89. Caldwell K, Baime M \& Wolever R (2012) Mindfulness based approaches to obesity and weight loss maintenance. $J$ Ment Health Couns 34, 269-282.

90. Lutz J, Herwig U, Opialla S, et al. (2014) Mindfulness and emotion regulation - an fMRI study. Soc $\operatorname{Cog} n$ Affect Neurosci 9, 776-785.

91. Murakami H, Katsunuma R, Oba K, et al. (2015) Neural networks for mindfulness and emotion suppression. PLOS ONE 10, e0128005.

92. Hölzel BK, Carmody J, Vangel M, et al. (2011) Mindfulness practice leads to increases in regional brain gray matter density. Psychiatry Res 191, 36-43.

93. Forman EM \& Butryn ML (2015) A new look at the science of weight control: how acceptance and commitment strategies can address the challenge of self-regulation. Appetite 84, 171-180.

94. Wansink B (2010) From mindless eating to mindlessly eating better. Physiol Behav 100, 454-463.

95. Kilpatrick LA, Suyenobu BY, Smith SR, et al. (2011) Impact of mindfulness-based stress reduction training on intrinsic brain connectivity. NeuroImage 56, 290-298.

96. Higgs S (2015) Manipulations of attention during eating and their effects on later snack intake. Appetite 92, 287-294.

97. Robinson E, Kersbergen I \& Higgs S (2014) Eating 'attentively' reduces later energy consumption in overweight and obese females. Br J Nutr 112, 657-661. 